\title{
UNIMODULARITY OF FINITE DIMENSIONAL HOPF ALGEBRAS
}

By

\author{
Satoshi SUZUKI
}

\section{Introduction.}

D. Radford [4] proved that a finite dimensional Hopf algebra $A$ is a symmetric algebra if and only if

(1) $A$ is unimodular, and

(2) the square of the antipode $s^{2}$ is an inner algebra-automorphism.

It is well-known that the 4-dimensional Hopf algebra of Sweedler shows that Condition (2) does not necessarily imply Condition (1).

We present in this paper an example which shows that the converse does not hold either.

\section{The construction.}

Let $n$ be a positive integer and $m_{i}$ an integer $\geqq 2$ for $1 \leqq i \leqq n$. Let $k$ be a field that contains a primitive $m_{i}$ th root of unity $\eta_{i}$ and let $\omega \in k$ be an element satisfying $\omega^{m_{i}}=1$. We may assume that $\omega$ is a primitive $m$ th root of unity for some positive integer $m$. We note that $m$ divides $m_{i}$.

As a general case we shall construct a Hopf algebra $B$ over $k$, which is generated as an algebra by $g_{i}, x_{i}$ subject to the relations;

$$
\begin{aligned}
& g_{j} g_{i}=g_{i} g_{j}, \quad g_{k}^{m_{k}}=1, \quad x_{k}^{m_{k}}=0, \quad x_{j} g_{i}=\omega g_{i} x_{j}, \\
& x_{k} g_{k}=\eta_{k} g_{k} x_{k}, \quad x_{i} g_{j}=\omega^{-1} g_{j} x_{i}, \quad x_{j} x_{i}=\omega x_{i} x_{j}, \quad \text { for } 1 \leqq k \leqq n, \quad 1 \leqq i \lessgtr j \leqq n .
\end{aligned}
$$

First, let $F=k\left[G_{1}, \cdots, G_{n}, G_{1}^{-1}, \cdots, G_{n}^{-1}, X_{1}, \cdots, X_{n}\right]$ be the free algebra on $3 n$ noncommuting indetarminates. We form the so-called free Hopf algebra

$$
F=k\left[G_{1}, \cdots, G_{n}, G_{1}^{-1}, \cdots, G_{n}^{-1}, X_{1}, \cdots, X_{n}\right] /\left(G_{i} G_{i}^{-1}-1, G_{i}^{-1} G_{i}-1\right) .
$$

The coalgebra structure maps $F \rightarrow F \otimes F$ and $F \rightarrow k$ are the algebrahomomorphisms determined by

$$
G_{i} \mapsto G_{i} \otimes G_{i}, \quad X_{i} \mapsto X_{i} \otimes G_{i}+1 \otimes X_{i},
$$

Received June 6, 1994. Revịsed October 28, 1994. 
and

$$
G_{i} \mapsto 1, \quad X_{i} \mapsto 0
$$

respectively. The antipode is the anti-algebra-homomorphism determined by

$$
G_{i} \mapsto G_{i}^{-1}, \quad X_{i} \mapsto-X_{i} G_{i}^{-1}
$$

Second, let $L$ be the ideal of $F$ generated by the following six subsets of $F$ :

(1) $\left\{G_{j} G_{i}-G_{i} G_{j} \mid 1 \leqq i \lesseqgtr j \leqq n\right\}$

(2) $\left\{G_{k}^{m_{k}}-1 \mid 1 \leqq k \leqq n\right\}$

(3) $\left\{X_{k}^{m_{k}} \mid 1 \leqq k \leqq n\right\}$

(4) $\left\{X_{j} G_{i}-\omega G_{i} X_{j}, X_{i} G_{j}-\omega^{-1} G_{j} X_{i} \mid 1 \leqq i \leqq j \leqq n\right\}$

(5) $\left\{X_{k} G_{k}-\eta_{k} G_{k} X_{k} \mid 1 \leqq k \leqq n\right\}$

(6) $\left\{X_{j} X_{i}-\omega X_{i} X_{j} \mid 1 \leqq i \leq j \leqq n\right\}$.

Let $B$ indicate $F / L$. Then denoting cosets by the small case letters, we have the multiplicative relations stated above.

Finally, we show the following proposition.

Proposition 1. (a) The ideal $L$ is a Hopf ideal of $F$, so $B$ is the Hopf algebra.

(b) $B$ has as a basis the set $\left\{g_{1}^{p_{1}} \cdots g_{n}^{p_{n}} \cdot x_{1}^{q_{1}} \cdots x_{n}^{q_{n}} \mid 0 \leqq p_{i}, q_{i} \leqq m_{i}-1\right\}$. Hence $\operatorname{dim} B=\Pi m_{i}^{2}$.

(c) The antipode $s$ of $B$ is given by

$$
s\left(g_{i}\right)=g_{i}^{-1}, \quad s\left(x_{i}\right)=-x_{i} g_{i}^{-1}=-\eta_{i}^{-1} g_{i}^{-1} x_{i}
$$

Hence $s^{2}\left(x_{i}\right)=\eta_{i}^{-1} x_{i}$ and the order of $s$ is (L.C.M. of $\left.\left\{m_{i}\right\}\right) \times 2$.

Proof. (a) We note that in a Hopf algebra an ideal generated by skew primitives is a Hopf ideal.

One can see that the elements of the above sets (1), (2) and (5) are all skew primitives. Thus the ideal $I, J$ and $K$ generated by (1), (2) and (5), respectively, are Hopf ideals.

Since the elements of the set (4) are skew primitives modulo $I$, the ideal $I^{\prime}$ generated by these, namely $I$ and (4), is a Hopf ideal; and since the elements of (6) are skew primitives modulo $I^{\prime}$, similarly the ideal $I^{\prime \prime}$ generated by these is also a Hopf ideal.

On the other hand, the elements of (3) are skew primitives modulo $K$ (c.f. [2, 
Lem. 2.5.], [6]). Thus the ideal $K^{\prime}$ generated by the two sets $K$ and (3) is a Hopf ideal.

Therefore the sum $I^{\prime \prime}+J+K^{\prime}$ is a Hopf ideal, and is just $L$.

(b) For $1 \leqq i \leqq n$ let $k\left[H_{i}\right], k\left[Y_{i}\right]$ be the polynomial algebras on 1 indeterminate $H_{i}, Y_{i}$, respectively, over $k$. Set $T_{i}=k\left[H_{i}\right] /\left(H_{i}^{m_{i}}-1\right), U_{i}=k\left[Y_{i}\right] /\left(Y_{i}^{m_{i}}\right)$, and $M=T_{1} \otimes \cdots \otimes T_{n} \otimes U_{1} \otimes \cdots \otimes U_{n}$. Denoting cosets by the small case letters, we see that $T_{i}$ has a basis $\left\{h_{i}^{j} \mid 0 \leqq j \leqq m_{i}-1\right\}$ and $U_{i}$ has a basis $\left\{y_{i}^{j} \mid 0 \leqq j \leqq m_{i}-1\right\}$. Therefore $M$ has the basis $\left\{h_{1}^{p_{1}} \otimes \cdots \otimes h_{n}^{p_{n}} \otimes y_{1}^{q_{1}} \otimes \cdots \otimes y_{n}^{q_{n}} \mid 0 \leqq p_{i}, q_{i} \leqq m_{i}-1\right\}$.

We define a left $B$-module structure on $M$.

First of all, we define an algebra-homomorphism from the free Hopf algebra $F$ to $\operatorname{End}_{k}(M)$, the algebra of endomorphisms of $M$, as follows: for $M \ni m=$ $h_{1}^{p_{1}} \otimes \cdots \otimes h_{n}^{p_{n}} \otimes y_{1}^{q_{1}} \otimes \cdots \otimes y_{n}^{q_{n}} \quad\left(0 \leqq p_{i}, q_{i} \leqq m_{i}-1\right)$,

$$
\begin{aligned}
& G_{i} \cdot m=h_{1}^{p_{1}} \otimes \cdots \otimes h_{i}^{p_{i}+1} \otimes \cdots \otimes h_{n}^{p_{n}} \otimes y_{1}^{q_{1}} \otimes \cdots \otimes y_{n}^{q_{n}}, \\
& X_{i} \cdot m=\omega^{p_{1}+\cdots+p_{i-1}} \eta_{i}^{p_{i}} \omega^{-\left(p_{i+1}+\cdots+p_{n}\right)} \omega^{q_{1}+\cdots+q_{i-1}} h_{1}^{p_{1}} \otimes \cdots \otimes h_{n}^{p_{n}} \otimes y_{1}^{q_{1}} \otimes \cdots \otimes y_{i}^{q_{i}+1} \otimes \cdots \otimes y_{n}^{q_{n}},
\end{aligned}
$$

but $p_{0}=q_{0}=0$. It is well-defined.

Next, we must show that the algebra-homomorphism annihilates the ideal $L$. For that purpose, it suffices to check on the six sets generating $L$.

On the sets (1), (2) and (3), it is clear.

On the set (4) for $1 \leqq i \lessgtr j \leqq n$,

$$
\begin{aligned}
X_{j} G_{i} \cdot m & =X_{j} \cdot h_{1}^{p_{1}} \otimes \cdots \otimes h_{i}^{p_{i}+1} \otimes \cdots \otimes h_{n}^{p_{n}} \otimes y_{1}^{q_{1}} \otimes \cdots \otimes y_{n}^{q_{n}} \\
& =\omega^{p_{1}+\cdots+p_{j-1}+1} \eta_{j}^{p_{j}} \omega^{-\left(p_{j+1}+\cdots+p_{n}\right)} \omega^{q_{1}+\cdots+q_{j-1}} \\
& h_{1}^{p_{1}} \otimes \cdots \otimes h_{i}^{p_{i}+1} \otimes \cdots \otimes h_{n}^{p_{n}} \otimes y_{1}^{q_{1}} \otimes \cdots \otimes y_{j}^{q_{j}+1} \otimes \cdots \otimes y_{n}^{q_{n}}, \\
G_{i} X_{j} \cdot m & =G_{i} \cdot\left(\omega^{p_{1}+\cdots+p_{j-1}} \eta_{j}^{p_{j}} \omega^{-\left(p_{j+1}+\cdots+p_{n}\right)} \omega^{q_{1}+\cdots+q_{j-1}}\right. \\
& \left.h_{1}^{p_{1}} \otimes \cdots \otimes h_{n}^{p_{n}} \otimes y_{1}^{q_{1}} \otimes \cdots \otimes y_{j}^{q_{j}+1} \otimes \cdots \otimes y_{n}^{q_{n}}\right) \\
& =\omega^{p_{1}+\cdots+p_{j-1}} \eta_{j}^{p_{j}} \omega^{-\left(p_{j+1}+\cdots+p_{n}\right)} \omega^{q_{1}+\cdots+q_{j-1}} \\
& h_{1}^{p_{1}} \otimes \cdots \otimes h_{i}^{p_{i}+1} \otimes \cdots \otimes h_{n}^{p_{n}} \otimes y_{1}^{q_{1}} \otimes \cdots \otimes y_{j}^{q_{j}+1} \otimes \cdots \otimes y_{n}^{q_{n}} .
\end{aligned}
$$

Thus

$$
\left(X_{j} G_{i}-\omega G_{i} X_{j}\right) \cdot M=(0)
$$

Similarly,

$$
\begin{aligned}
X_{i} G_{j} \cdot m & =X_{i} \cdot h_{1}^{p_{1}} \otimes \cdots \otimes h_{j}^{p_{j}+1} \otimes \cdots \otimes h_{n}^{p_{n}} \otimes y_{1}^{q_{1}} \otimes \cdots \otimes y_{n}^{q_{n}} \\
& =\omega^{p_{1}+\cdots+p_{i-1}} \eta_{i}^{p_{i}} \omega^{-\left(p_{i+1}+\cdots+p_{n}+1\right)} \omega^{q_{1}+\cdots+q_{i-1}}
\end{aligned}
$$




$$
\begin{gathered}
h_{1}^{p_{1}} \otimes \cdots \otimes h_{j}^{p_{j}+1} \otimes \cdots \otimes h_{n}^{p_{n}} \otimes y_{1}^{q_{1}} \otimes \cdots \otimes y_{i}^{q_{i}+1} \otimes \cdots \otimes y_{n}^{q_{n}}, \\
G_{j} X_{i} \cdot m=G_{j} \cdot\left(\omega^{p_{1}+\cdots+p_{i-1}} \eta_{i}^{p_{i}} \omega^{-\left(p_{i+1}+\cdots+p_{n}\right)} \omega^{q_{1}+\cdots+q_{i-1}}\right. \\
\left.h_{1}^{p_{1}} \otimes \cdots \otimes h_{n}^{p_{n}} \otimes y_{1}^{q_{1}} \otimes \cdots \otimes y_{i}^{q_{i}+1} \otimes \cdots \otimes y_{n}^{q_{n}}\right) \\
=\omega^{p_{1}+\cdots+p_{i-1}} \eta_{i}^{p_{i}} \omega^{-\left(p_{i+1}+\cdots+p_{n}\right)} \omega^{q_{1}+\cdots+q_{i-1}} \\
h_{1}^{p_{1}} \otimes \cdots \otimes h_{j}^{p_{j}+1} \otimes \cdots \otimes h_{n}^{p_{n}} \otimes y_{1}^{q_{1}} \otimes \cdots \otimes y_{i}^{q_{i}+1} \otimes \cdots \otimes y_{n}^{q_{n}} .
\end{gathered}
$$

Thus

$$
\left(X_{i} G_{j}-\omega^{-1} G_{j} X_{i}\right) \cdot M=(0)
$$

Therefore the algebra-homomorphism annihilates the set (4).

In a similar fashion, one can check that it takes zero on the sets (5), (6).

Hence the map annihilates the ideal $L$, so $M$ can be seen as a left $B$-module.

Define a linear map $\phi: M \rightarrow B$ by $h_{1}^{p_{1}} \otimes \cdots \otimes h_{n}^{p_{n}} \otimes y_{1}^{q_{1}} \otimes \cdots \otimes y_{n}^{q_{n}} \mapsto g_{1}^{p_{1}} \cdots g_{n}^{p_{n}}$ $\cdot x_{1}^{q_{1}} \cdots x_{n}^{q_{n}}$. It is easy to see that $\phi$ is surjective. Define a $B$-module map $\psi: B \rightarrow M$ by $a \mapsto a \cdot 1 \otimes \cdots \otimes 1$. Then $\psi \circ \phi$ is the identity map, hence $\phi$ is bijective.

Therefore the set of the statement is a basis of $B$.

(c) It is clear.

This completes the proof of the proposition.

In this way we can form the Hopf algebra $B$, which is a generalization of $[3$, 4.4].

Now we shall notice the unimodularity. A Hopf algebra is called unimodular if the left integral space and the right one are the same (c.f. [3]).

LEMMA 2. (a) Set $\Lambda=\left(1+g_{1}+\cdots+g_{1}^{m_{1}-1}\right) \cdots\left(1+g_{n}+\cdots+g_{n}^{m_{n}-1}\right) \cdot x_{1}^{m_{1}-1} \cdots x_{n}^{m_{n}-1}$. Then $\Lambda$ is a non-zero left integral.

(b) $B$ is unimodular if and only if $\eta_{i}=\omega^{-n+2 i-1}$ for $1 \leqq i \leqq n$.

Let $B$ be unimodular, then we have

(c) $n$ is even,

(d) $m=m_{i}$ for $1 \leqq i \leqq n$, i.e. $\eta_{i}$ is a primitive mth root of unity,

(d') $(n-2 i+1, m)=1$, i.e. $n-2 i+1$ and $m$ are relatively prime, for $1 \leqq i \leqq n$,

(d") $(p, m)=1$ for each odd prime $p \leqq n-1$,

(e) $s^{2}\left(x_{i}\right)=\eta_{i}^{-1} x_{i}=\omega^{n-2 i+1} x_{i}$.

Proof. (a) Clear.

(b) It follows that $\Lambda g_{i}=\omega^{-n+2 i-1} \eta_{i}^{-1} \Lambda$ and $\Lambda x_{i}=0$. Indeed, $\Lambda g_{i}=$ 
$\omega^{-(n-i)} \eta_{i}^{-1} \omega^{i-1} \Lambda=\omega^{-n+2 i-1} \eta_{i}^{-1} \Lambda$. Hence $B$ is unimodular if and only if $\eta_{i}=\omega^{-n+2 i-1}$ for $1 \leqq i \leqq n$.

(c) Suppose that $n$ is odd. Then by (b) $\eta_{\frac{n+1}{2}}=\omega^{0}=1$, a contradiction.

(d) Recall that $m$ divides $m_{i}$ for all $i$. Since $\eta_{i}^{m}=1$ holds by (b) and $\eta_{i}$ is a primitive $m_{i}$ th root of unity, $m_{i}$ divides $m$. Hence $m=m_{i}$.

(d') This follows from (b) and (d).

(d") This follows from (c) and (d').

(e) By (b) and Proposition 1.(c).

This completes the proof of the lemma.

We note that (d), (d') and (d") are equivalent under the conditions $\eta_{i}=\omega^{-n+2 i-1}$ and (c), and notice that Lemma 2 shows us the way of direct construction of unimodular Hopf algebra $B$, that is;

PROPOSITION 3. Suppose that integers $n$ and $m \geqq 2$ satisfy the conditions (c), (d") in Lemma 2. For a primitive mth root of unity $\omega$, set $\eta_{i}=\omega^{-n+2 i-1}$ (Note that this is a primitive mth root of unity). Then the Hopf algebra $B$ is unimodular.

Let $B_{n, m}$ indicate $B$ in case of Proposition 3.

\section{The relation between the unimodularity and $s^{2}$}

We only consider the unimodular Hopf algebra $B_{n, m}$ and show that Condition (1) does not necessarily imply Condition (2).

LEMMA 4. Suppose in addition that $n \geqq 4$ and $m$ is divided by 4 . Let $\mu=\frac{m}{4}$ and. $v=\frac{n}{2}$, set $z=x_{v-1}^{\mu}+x_{v}^{\mu} \in B_{n, m}$ (Note that neither $x_{v-1}^{\mu}$ nor $x_{v}^{\mu}$ is zero, so $\mathrm{z} \neq$ $0)$. Then there is an element $\zeta_{i} \in k$ such that $z g_{i}=\zeta_{i} g_{i} z$ for $1 \leqq i \leqq n$.

ProOF.

It is easy to see that the statement is true for $1 \leqq i \leqq v-2$ or $v+1 \leqq i \leqq n$.

i) $i=v-1$ :

$$
\begin{aligned}
x_{v-1}^{\mu} g_{v-1} & =\eta_{v-1}^{\mu} g_{v-1} x_{v-1}^{\mu} \\
& =\omega^{-3 \mu} g_{v-1} x_{v-1}^{\mu} \quad\left(\text { since } \eta_{v-1}=\omega^{-3}\right) \\
& =\omega^{\mu} g_{v-1} x_{v-1}^{\mu}, \\
x_{v}^{\mu} g_{v-1} & =\omega^{\mu} g_{v-1} x_{v}^{\mu} .
\end{aligned}
$$




$$
x_{v}^{\mu} g_{v-1}=\omega^{\mu} g_{v-1} x_{v}^{\mu} .
$$

Thus it follows that $z g_{v-1}=\omega^{\mu} g_{v-1} z$, so set $\zeta_{v-1}=\omega^{\mu}$.

ii) $i=v$ :

$$
\begin{aligned}
x_{v-1}^{\mu} g_{v} & =\omega^{-\mu} g_{v} x_{v-1}^{\mu}, \\
x_{v}^{\mu} g_{v} & =\eta_{v}^{\mu} g_{v} x_{v}^{\mu} \\
& =\omega^{-\mu} g_{v} x_{v}^{\mu} . \quad\left(\text { since } \eta_{v}=\omega^{-1}\right) .
\end{aligned}
$$

Thus it follows that $z g_{v}=\omega^{-\mu} g_{v} z$, so set $\zeta_{v}=\omega^{-\mu}$.

This completes the proof.

TheOREM 5. Let $n, m$ be as in Proposition 3. If $n \geqq 4$ and $m$ is divided by 4 , the square of antipode $s^{2}$ of $B_{n, m}$ is not inner.

Proof. Let $z, \mu$ and $v$ be as in Lemma 4. It follows that

$$
s^{2}(z)=\omega^{3 \mu} x_{v-1}^{\mu}+\omega^{\mu} x_{v}^{\mu} .
$$

Now suppose that $s^{2}(z)=a z b$ for some $a, b \in B_{n, m}$.

Write

$$
\begin{aligned}
& a=\sum \alpha_{p_{1} \cdots p_{n} q_{1} \cdots q_{n}} g_{1}^{p_{1}} \cdots g_{n}^{p_{n}} \cdot x_{1}^{q_{1}} \cdots x_{n}^{q_{n}}, \\
& \text { where } \quad \alpha_{p_{1} \cdots p_{n} q_{1} \cdots q_{n}} \in k, 0 \leqq p_{i}, q_{i} \leqq m-1, \\
& b=\sum \beta_{p_{1}^{\prime} \cdots p_{n}^{\prime} q_{1}^{\prime} \cdots q_{n}^{\prime}} g_{1}^{p_{1}^{\prime}} \cdots g_{n}^{p_{n}^{\prime}} \cdot x_{1}^{q_{1}^{\prime}} \cdots x_{n}^{q_{n}^{\prime}}, \\
& \text { where } \quad \beta_{p_{1}^{\prime} \cdots p_{n}^{\prime} q_{1}^{\prime} \cdots q_{n}^{\prime}} \in k, 0 \leqq p_{n}^{\prime}, q_{n}^{\prime} \leqq m-1,
\end{aligned}
$$

and divide $a$ into $a_{0}+a_{1}$, where $a_{0}$ is the part of $q_{i}=0$ and $a_{1}$ is the rest, $b$ into $b_{0}+b_{1}$, where $b_{0}$ is the part of $q_{i}^{\prime}=0$ and $b_{1}$ is the rest.

Then

$$
\begin{aligned}
a z b= & \left(a_{0}+a_{1}\right) z\left(b_{0}+b_{1}\right) \\
= & a_{0} z b_{0}+a_{0} z b_{1}+a_{1} z b_{0}+a_{1} z b_{1} \\
= & a_{0} z b_{0}+w, \text { where } w=a_{0} z b_{1}+a_{1} z b_{0}+a_{1} z b_{1}, \\
= & c_{0} z+w, \\
& \quad \text { where } c_{0}=\sum \gamma_{r_{1} \cdots r_{n}} g_{1}^{r_{1}} \cdots g_{n}^{r_{n}}, \quad \gamma_{r_{1} \cdots r_{n}} \in k, 0 \leqq r_{i} \leqq m-1, \\
& \quad \text { by Lemma 4, } \\
= & c_{0} x_{v-1}^{\mu}+c_{0} x_{v}^{\mu}+w .
\end{aligned}
$$


So $s^{2}(z)=a z b$ implies

$$
\omega^{3 \mu} x_{v-1}^{\mu}+\omega^{\mu} x_{v}^{\mu}=c_{0} x_{v-1}^{\mu}+c_{0} x_{v}^{\mu}+w .
$$

Thus $w=0$ since $w$ is a sum of terms that contain at least $\mu+1 x$ 's as factors, and $c_{0}=\gamma_{0 \cdots 0} \cdot 1$ since $\gamma_{r_{1} \ldots r_{n}}=0$ if some $r_{i} \neq 0$. Set $\gamma=\gamma_{0 \cdots 0}$. Then

$$
\omega^{3 \mu} x_{v-1}^{\mu}+\omega^{\mu} x_{v}^{\mu}=\gamma x_{v-1}^{\mu}+\gamma x_{v}^{\mu} .
$$

Again comparing the coefficients, we have $\omega^{3 \mu}=\gamma=\omega^{\mu}$, so $\omega^{(3 \mu-\mu)}=\omega^{2 \mu}=$ $\omega^{\frac{m}{2}}=1$, a contradiction since $\omega$ is a primitive $m$ th root of unity.

Therefore for any $a, b \in B_{n, m}, s^{2}(z) \neq a z b$. This implies that $s^{2}$ cannot be inner.

This completes the proof.

REMARKS. For another $B_{n, m}$, we have the following:

(1) For any $m$, the square of antipode $s^{2}$ of $B_{2, m}([3,4.4])$ is inner. More precisely,

$$
s^{2}(?)=\left(g_{1}^{p} g_{2}^{q}\right)^{-1} \cdot ? \cdot\left(g_{1}^{p} g_{2}^{q}\right),
$$

where $p, q$ are integers such that $p+q \equiv-1$ modulo $m$.

(2) Suppose that $n \geqq 4$ and either that $m$ is odd or that $m$ is even but is not divided by 4 . Then the square of antipode of $B_{n, m}$ is inner as follows.

Fix an integer $l$ such that $m=2 l+1\left(\frac{m}{2}=2 l+1\right.$, resp.). Set $g=g_{1}^{l} \cdots g_{\frac{n}{2}-1}^{l} \cdot g_{\frac{n}{2}}^{p}$. $g_{\frac{n}{2}+1}^{q} \cdot g_{\frac{n}{2}+2}^{l} \cdots g_{n}^{l}$, where $p$ and $q$ are integers such that $p+q \equiv-1$ modulo $m$. Then

$$
s^{2}(?)=g^{-1} \cdot ? \cdot g
$$

(3) In general if both the dimension of a Hopf algebra and the order of the square of antipode $s^{2}$ are odd, then $s^{2}$ is inner [1, Prop. 1].

Therefore we have the following;

THEOREM 6. There exists a finite dimensional unimodular Hopf algebra such that the square of antipode is not inner.

\section{Ref erences}

[1] Kauffman, L. H., Radford, D. E., A necessary and sufficient condition for a finite-dimensional Drinfel'd double to be a ribbon Hopf algebra, J. Algebra 159 (1993), 98-114.

[2] Masuoka, A., Cleft extensions for a Hopf algebra generated by a nearly primitive element, Comm. Algebra 22 (1994), 4537-4559. 
[3] Radford, D. E., The order of the antipode of a finite dimensional Hopf algebra is finite, Amer. J. Math. 98 (1976), 333-355.

[4] Radford, D. E., The trace function and Hopf algebras, J. Algebra 163 (1994), 583-622.

[5] Sweedler, M., Hopf Algebras, Benjamin, New York, 1969.

[6] Taft, E. J., The order of the antipode of finite-dimensional Hopf algebra, Proc. Nat. Acad. Sci. USA 68 (1971), 2631-2633.

\author{
Institute of Mathematics, \\ University of Tsukuba, \\ Ibaraki 305 Japan
}

